\title{
A Numerical Investigation of the Characteristics of Porous Vacuum Preloaded Air Bearings
}

\author{
K. Ermis ${ }^{1}$ and R. Sener ${ }^{2 \dagger}$ \\ ${ }^{1}$ Mechanical Engineering, Technology Faculty, Sakarya University of Applied Science, Esentepe Campus, \\ 54050, Sakarya, Turkey \\ ${ }^{2}$ Automotive Engineering, Technology Faculty, Batman University, 72100, Batman, Turkey \\ †Corresponding Author Email: ramazan.sener@batman.edu.tr
}

(Received December 4, 2020; accepted April 6, 2021)

\begin{abstract}
Air bearings are widely used in devices that require high-precision. Vacuum preloading is a compact solution, as it does not add extra weight to the air bearing. In addition, the usage of porous material ensures that the pressure is distributed homogeneously to the air film. In this study, a numerical analysis was performed using three vacuum preloaded porous air bearings with different diameters $(50,75$, and $90 \mathrm{~mm})$. The effect of applied tensile and compression loads on the air film thickness of the air bearings was determined using experimental methods. It was found that air film thickness increases with increasing tensile load and decreases with increasing compressive load. CFD model parameters were set to be the same as those for experimental conditions. It was concluded that the CFD model is consistent with experimental results. The pressure distribution in the air film and the load-carrying capacity of the air bearings were obtained using the CFD simulation.
\end{abstract}

Keywords: Air bearing; Porous; Vacuum preloaded; CFD; Load-carrying capacity.

\section{NOMENCLATURE}

\begin{tabular}{|c|c|c|c|}
\hline 1D & one-dimensional & $\mathrm{S}$ & source term \\
\hline $3 \mathrm{D}$ & three-dimensional & $\mathrm{S}_{\mathrm{a}}$ & surface area of the air film \\
\hline B-50mm & $50 \mathrm{~mm}$ diameter air bearing & $\mathrm{v}$ & velocity \\
\hline $\mathrm{B}-75 \mathrm{~mm}$ & $75 \mathrm{~mm}$ diameter air bearing & VPL & vacuum preloaded \\
\hline B-90mm & $90 \mathrm{~mm}$ diameter air bearing & $\mathrm{y}^{+}$ & dimensionless distance to the wall \\
\hline CAD & Computer Aided Design & W & load-carrying capacity \\
\hline CFD & Computational Fluid Dynamics & $\beta$ & model coefficient \\
\hline CPU & Central Processing Unit & $\mathrm{cf}_{\mathrm{f}}$ & Forchheimer or Ergün constant \\
\hline DP & spherical particle diameter & $\varepsilon$ & dissipation rate \\
\hline F & force & $\mathrm{k}$ & turbulent kinetic energy \\
\hline $\mathrm{F}_{2}$ & blending function & $\mu$ & dynamic viscosity of fluid \\
\hline h & air film thickness & $\rho$ & density \\
\hline K & permeability & $\sigma_{\mathrm{k}}$ & turbulent Prandtl numbers for $\mathrm{k}$ \\
\hline$P$ & pressure & $\sigma_{\omega}$ & turbulent Prandtl numbers for $\omega$ \\
\hline $\mathrm{R}$ & stiffness & $\omega$ & specific dissipation rate \\
\hline
\end{tabular}

\section{INTRODUCTION}

Bearings are one of the age-old problems for engineers. Numerous machines use bearings to support their movement. Bearings are machine elements that allow the movement of components relative to each other. Plain or Rolling-element bearings have mechanical contact between their

components. Mechanical contact decreases contact fatigue life (Liu 2020; Liu and Shao 2018). Fluid bearings are non-contact bearings where the load is supported by a gas or liquid. Owing to the absence of mechanical contact and static friction, they can have an almost infinite lifetime. As the precision, resolution, and speed of the machines increase, the limits of the bearing technology are challenged. 
Therefore, there is a need for air bearings. Aerostatic or air bearings provide very low to almost zero friction using pressurized air. There are very high precision air bearings; the movement accuracy of which does not exceed $10 \mathrm{~nm}$. These bearings are used in very high-precision, optical quality surfaces, and ultra-precision machining applications (Miyatake and Yoshimoto 2010; Wardle 2015).

Air bearings operate with an external high-pressure air source to create load-bearing capacity. In most air bearing applications, air pressure is around 400 to $600 \mathrm{kPa}$, which is what is typically used in the industry. As the inlet pressure is relatively small, the load-carrying capacity is limited. In critical applications, air bearings can be operated with up to $1000 \mathrm{kPa}$ pressure using a special compressor. This improves stiffness and load-carrying capacity (Wardle 2015). The air bearing has prominent advantages such as extremely low friction, low wear, and high speed resolution over alternative bearing concepts (Bleuler et al. 2000; Gao et al. 2019). The air bearing is used as a component that facilitates the design and assembly of ultra-precise equipment. It is highly successful in absorbing environmental vibrations (Torralba et al. 2016).

The main disadvantage of air bearings is that there is a very small gap between the components and the bearing. Accordingly, it requires very tight tolerances. It needs pressurized air constantly during its operation. Also, air bearings have low stiffness. However, stiffness can be significantly increased by preloading the bearing. There are four common methods for preloading air bearings such as weight adding, magnetic attraction, opposed assembly, and vacuum preloading. The vacuum preloading method is widely used since it does not add extra weight and hence is compact (Khim and Park 2013; Ye et al. 2009). A porous material is used to distribute the air homogeneously to the contact surface and to achieve homogeneous pressure distribution in the air bearing (Kwan and Corbett 1998; Schenk et al. 2008).

Vacuum preloaded (VPL) porous air bearings provide frictionless motion and high stiffness. VPL air bearing uses a combination of air pressure and vacuum as shown in Fig. 1. Pressurized air prevents physical contact of the bearing with the guide while the vacuum under the bearing structure preloads the bearing on the guide surface. While the vacuum is preloading the bearing on the guide, pressurized air prevents physical contact of the bearing with the guide surface.

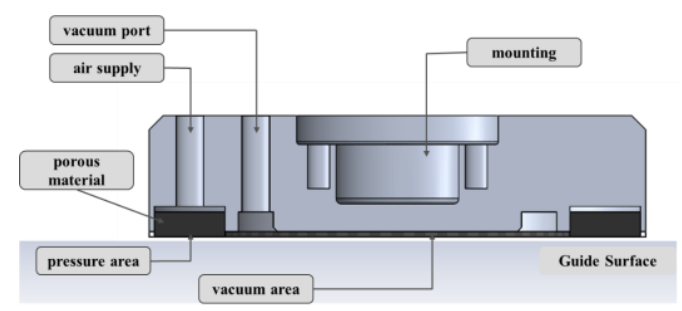

Fig. 1. Schematic view of the cross-section of the circular vacuum preloaded porous air bearing.
Singh and Rao conducted a theoretical analysis to determine the static properties of a circular porous air bearing. The analysis assumed that the flow through the porous material is axial. The pressure distribution inside the bearing was solved numerically with the modified Reynolds equation using the finite difference method. Various inlet pressures, bearing capacity, film thickness, and effects of orifice size were investigated. According to their analyses, the circular air bearing does not negatively affect the static performance from the slope. Furthermore, the orifice diameter significantly affects the load-capacity (Singh and Rao 1982). AI-Bender and Brussel studied the tilt characteristics of the circular center-fed air bearings. They developed a theoretical model and confirmed the model with experiments. According to their studies, the dimensionless tilt stiffness increases proportionally with the dimensionless load (Al-Bender and Van Brussel 1992). Fourka and Bonis analyzed the influence of the type of feeding system on the performance of air bearings. He compared load capacity, stiffness, airflow, porous, and orifice inlet designs. According to their studies, the number of orifices, and the orifice location are important when it comes to achieving optimum performance (Fourka and Bonis 1997). Yoshimoto and Kohno studied the dynamic and static properties of the aerostatic porous bearings. They examined the air supply technique of the aerostatic porous air bearing by experimental and theoretical methods. They determined that the maximum load capacity did not depend on the air supply area. The dimensionless static stiffness increased with the air inlet outer diameter.

They found that the restricted layer reduced the influence of the supply area shape on the bearing properties (Yoshimoto and Kohno 2001). Slocum et $a l$. used the carbon porous air bearing as the carrier, by designing a precise and simple linear axis. They assembled the air bearings by vacuuming between the two vertical plates, injecting epoxy between the carrier and the bearing. The control of the flatness tolerances of the bearings and planes leads to a high degree of accuracy and moment load capacity. Their design has gained good dynamic stiffness due to the large bearing area. Their system can operate quite steadily even when large external moments are applied. It is anticipated that this system can be considered as an alternative to ball-screw systems, taking into account total loads (Slocum et al. 2003). Plante et al. developed a design model for the circular porous air bearing using the onedimensional (1D) generalized flow method. They aimed to better understand the porous air bearing with the developed model. When they compared the 1D model results to the experimental results, the model had acceptable accuracy. Also, the 1D model of the air bearings helps to understand the physics of the fluid flow. The optimum design can be developed by performing parametric studies on various design parameters (Plante et al. 2005). Gao et al. used parametric computational fluid dynamics (CFD) models and a genetic algorithm to obtain optimum design parameters for an air bearing. They aimed to maximize the stiffness and load-bearing 
capacity of the air bearing. They evaluated the performance of the air bearing. Pareto optimum results were obtained using the CFD model and a multipurpose genetic algorithm. They found that the stiffness and the load-carrying capacity increased by $39 \%$ and $34 \%$, respectively. In addition, airflow decreased by $20 \%$. (Gao et al. 2018). Shi et al. studied the dynamic and static characteristics of air bearings in three degrees of freedom. They designed the model to achieve the damping and dynamic stiffness coefficients. They evaluated the effect of air film thickness, rotational speed, and pitch angles on the load-carrying capacity, dynamic stiffness, and load moments. They found that as the air film thickness increased, the load-carrying capacity and the load moment decreased. They determined that as the slope angle increases, the airflow decreases while the load capacity increases (Shi et al. 2019). Ishibashi et al. investigated the dynamic and static characteristics of the circular single orifice air bearing. The flow inside the air bearing was resolved using a CFD simulation, taking into account the inertial forces. They compared the CFD simulation and traditional Reynolds equation results with the experimental results. They found that the CFD simulation provides more accurate results than the traditional Reynolds equation. Dynamic stiffness and damping coefficient could be estimated numerically using CFD simulation techniques (Ishibashi et al. 2019).

According to the literature review, air bearings are of great importance for the industry. Especially, the use of porous and vacuum preloaded air bearings is becoming more common every day because of their homogeneous pressure distribution, low weight, and high stiffness. Theoretical and experimental studies on the modeling and characterization of air bearings are available in the literature (Aguirre et al. 2010; Li et al. 2018; Nishio et al. 2011; Oiwa et al. 2012). However, there is a gap in the literature especially regarding porous and vacuum preloaded air bearings. Investigation and characterization of fluid behavior in the bearing will contribute to a better understanding of VPL porous air bearings. Nevertheless, VPL air bearings are promising in metrology, precision machine tools, and semiconductors applications (Cai et al. 2011; Connolly et al. 2019; Gordon and Erkorkmaz 2012; Horiuchi and Suzuki 2013; Okyay et al. 2018; Rybus and Seweryn 2016). Vacuum preloaded air bearings will contribute to the development of precision machines with their compact dimensions and higher stiffness value. Hence, it is important to understand and characterize the properties of VPL porous air bearings. This study aims to characterize the VPL porous air bearing under static loading conditions and conduct a CFD simulation of the fluid flow inside the bearing. CFD modeling can lead to further improvement of the bearing's features.

\section{Methodology}

Experiments were conducted at the Waterloo University, Mechanical Engineering Department,
Precision Controls Laboratory (Ruszkowski et al. 2012). The experimental setup was designed to characterize the vacuum preloaded porous air bearing (Fig. 2). Displacements of the bearing during applied static load and moment were measured using capacitive sensor probes. VPL porous air bearings of New Way Precision were used in the experiments. Specifications of the three air bearings with varying diameters are presented in Table 1 (New Way Air Bearings 2006). Air bearings with three different diameters, $50 \mathrm{~mm} 75$ $\mathrm{mm}$, and $90 \mathrm{~mm}$, were tested (Devitt 1999). The bearing was supplied with pressurized air and then a loading array was applied using different weights. An equivalent unloading sequence was then followed, which allowed any hysteresis effects to be observed. The experiments were carried out with an input pressure of $410 \mathrm{kPa}$ and an input vacuum of $50.80 \mathrm{kPa}$.

Table 1 Specifications of VPL porous air bearings.

\begin{tabular}{|c|c|c|c|}
\hline & $\begin{array}{l}\Phi 50 \\
\mathrm{~mm}\end{array}$ & $\begin{array}{l}\Phi 75 \\
\mathrm{~mm}\end{array}$ & $\begin{array}{l}\Phi 90 \\
\mathrm{~mm}\end{array}$ \\
\hline $\begin{array}{l}\text { Input Pressure } \\
{[\mathrm{MPa}]}\end{array}$ & 0.41 & 0.41 & 0.41 \\
\hline $\begin{array}{l}\text { Input Vacuum } \\
\text { [in } \mathrm{Hg} \text { ] }\end{array}$ & 15 & 15 & 15 \\
\hline Ideal Load [N] & 45 & 110 & 150 \\
\hline Stiffness $[\mathrm{N} / \mu \mathrm{m}]$ & 13 & 29 & 43 \\
\hline Flow [NLPM] & 1.06 & 1.38 & 1.48 \\
\hline $\begin{array}{l}\text { Air film thickness } \\
{[\mu \mathrm{m}]}\end{array}$ & 5 & 5 & 5 \\
\hline $\begin{array}{l}\text { Bearing diameter } \\
{[\mathrm{mm}]}\end{array}$ & 50 & 75 & 90 \\
\hline $\begin{array}{l}\text { Bearing height } \\
{[\mathrm{mm}]}\end{array}$ & 22 & 22 & 22 \\
\hline $\begin{array}{l}\text { Bearing weight } \\
{[\mathrm{g}]}\end{array}$ & 73 & 203 & 306 \\
\hline Bearing material & \multicolumn{3}{|c|}{ Aluminum } \\
\hline $\begin{array}{l}\text { Porous media } \\
\text { material }\end{array}$ & \multicolumn{3}{|c|}{ Carbon } \\
\hline Resolution & \multicolumn{3}{|c|}{ Infinite } \\
\hline $\begin{array}{l}\text { Maximum speed } \\
{[\mathrm{m} / \mathrm{s}]}\end{array}$ & \multicolumn{3}{|c|}{50} \\
\hline $\begin{array}{l}\text { Guide surface } \\
\text { finish [RMS] }\end{array}$ & \multicolumn{3}{|c|}{16} \\
\hline
\end{tabular}

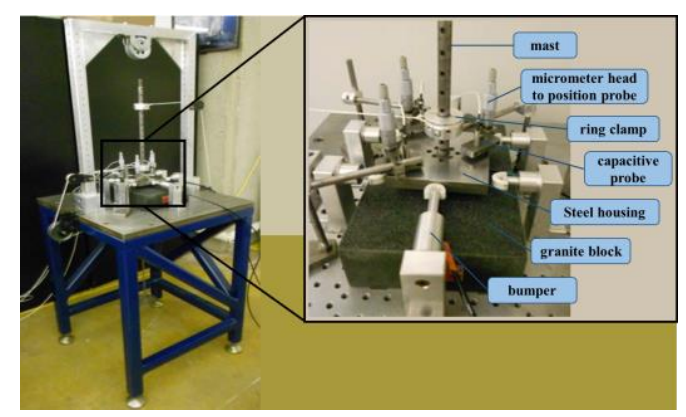

Fig. 2. A photographic view of the experimental setup. 


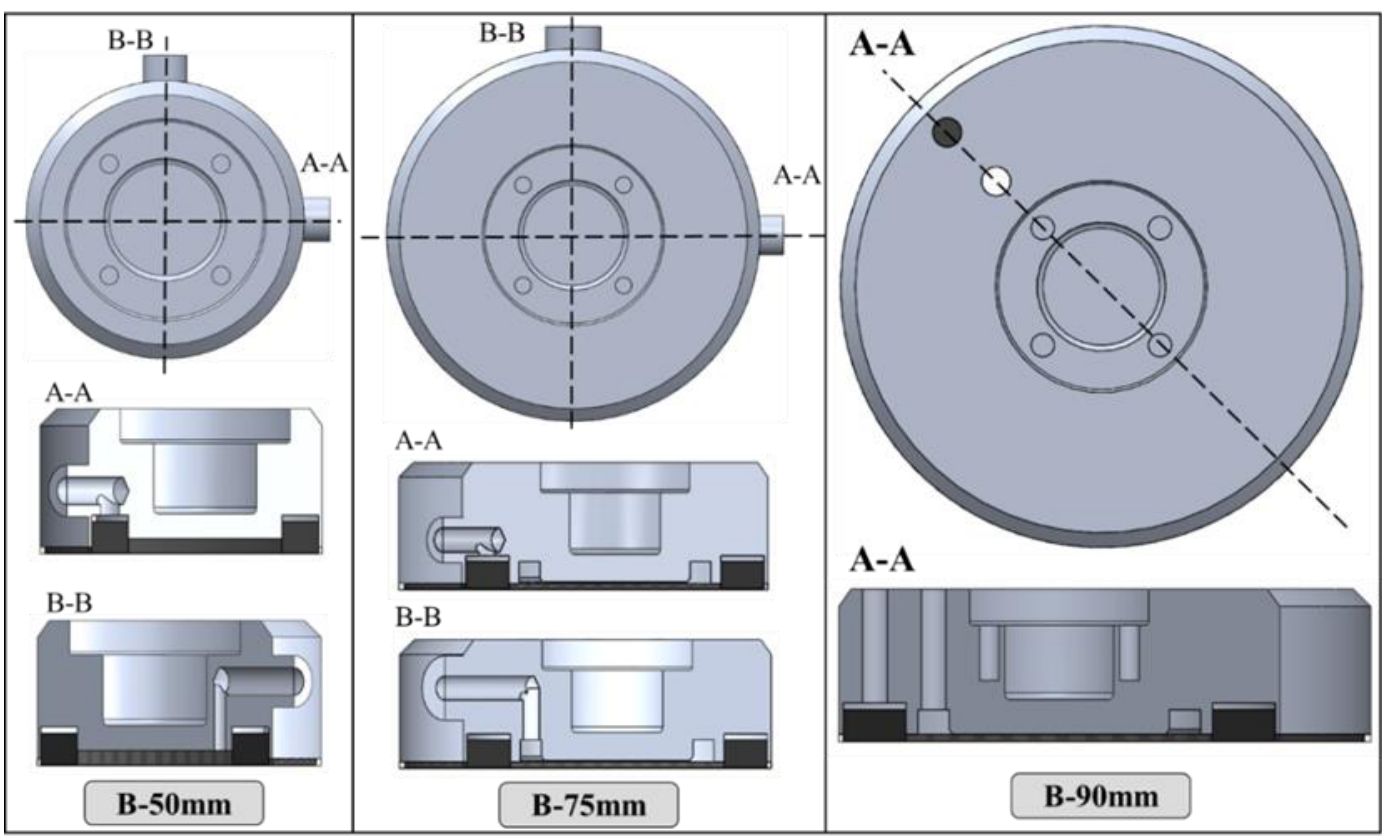

Fig. 3. CAD model of the VPL porous air bearing.
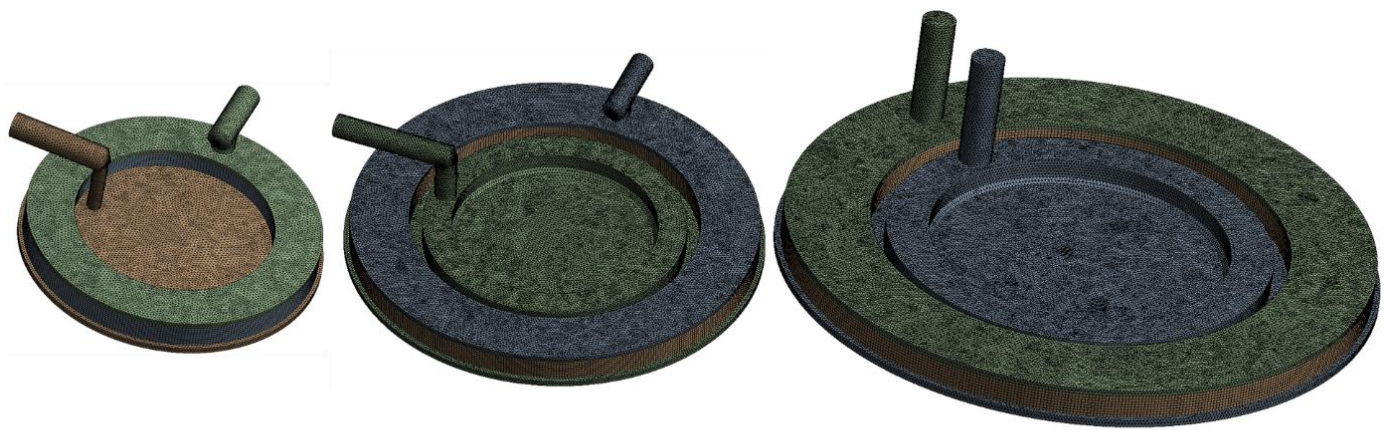

B-50mm

B-75mm

B-90mm

Fig. 4. Mesh of the fluid and porous part of the air bearings.

Three VPL porous air bearings were designed with different diameters for the 3D CFD simulation. Accordingly, the solid part of the bearing, the porous part, and the interior air part were generated (Fig. 3). Carbon was used for the porous material, and aluminum was used for the bearing material. Although the diameters of the bearings were different $(50,75$, and $90 \mathrm{~mm})$, the height values were fixed at $22 \mathrm{~mm}$.

There was only a small design difference between the three bearings. While the pressure and vacuum inlets were from the side for the bearings with a diameter of $50 \mathrm{~mm}$ and $75 \mathrm{~mm}$, these inlets were from the upper side for the bearing of $90 \mathrm{~mm}$ diameter.

Meshing is critical for numerical modeling. The convergence, reliability, and accuracy of the solution depend on the meshing. The mesh independent studies were performed for this simulation since the simulation results are sensitive to the grid size. $\mathrm{y}+$ is the dimensionless length associated with the mesh resolution near the wall. The distance to the boundary layer significantly affects the accuracy of the CFD solution. Variables of the solution have wide gradients close to the wall.

Therefore, it was proposed that the $y+$ value of the mesh should be below 5 (Xue et al. 2020). For the medium and fine mesh, the $\mathrm{y}+$ value has remained below 5 (Table 2).

As can be seen from Table 2, the refinement of the mesh does not significantly affect the simulation result for the medium and fine mesh. The difference between the fine and medium mesh results is about $1 \%$, but the fine mesh extends the CPU time considerably. In this study, it has been determined that the optimum mesh is medium with approximately 1 million elements. Similar mesh parameters were applied to three different designs (Fig. 4).

ANSYS $^{\circledR}$ Fluent commercial CFD code was used to solve the equations of the fluid flow inside the air 
bearings (ANSYS Inc, 2019). With the finite volume approach, this code is used for modeling single or multiphase fluid flow, heat transfer, and chemical reactions for complicated geometries. In the CFD simulation, the inlet pressure was set to $410 \mathrm{kPa}$, the inlet vacuum was set to $-50.8 \mathrm{kPa}$ and the barometric pressure was implemented to the air outlet. The other walls were defined as stationary and impermeable. The reference temperature and pressure were $293.15 \mathrm{~K}$ and $101.325 \mathrm{kPa}$ (the standard atmospheric pressure), respectively.

Table 2 Mesh independency results.

\begin{tabular}{|c|c|c|c|}
\hline Mesh & $\begin{array}{c}\text { Total } \\
\text { number }\end{array}$ & $\begin{array}{c}\text { Max } \\
\mathrm{y}^{+}[-]\end{array}$ & $\begin{array}{c}\text { Load-carrying } \\
\text { capacity [N] }\end{array}$ \\
\hline Course & 330,116 & 7.89 & 781.96 \\
\hline Medium & $1,017,603$ & 3.12 & 780.45 \\
\hline Fine & $4,049,240$ & 2.38 & 779.05 \\
\hline
\end{tabular}

The finite volume method was used and the governing equations of fluid flow are given below (Kakaç et al. 2007). Mass and momentum conservation equations are defined as follows:

$$
\frac{\partial \rho}{\partial t}+\nabla *(\rho \vec{v})=S_{M}
$$

$\frac{\partial}{\partial t}(\rho \vec{v})+\nabla *(\rho \overrightarrow{v v})$

$=-\nabla \mathrm{p}+\nabla *(\vec{\tau})+\rho \vec{g}+\vec{F}$

The energy equation is defined by the equation below:

$$
\begin{aligned}
& \frac{\partial}{\partial t}(\rho e)-\nabla *(\rho e \vec{v}) \\
& =\frac{\partial p}{\partial t}+\nabla *(k \nabla T)+S_{e}+S_{r a d}
\end{aligned}
$$

The k-omega SST turbulence model is defined by the following equations:

$$
\begin{aligned}
& \frac{\partial k}{\partial t}+U_{j} \frac{\partial k}{\partial x_{j}} \\
& =P_{k}-\beta^{*} k \omega+\frac{\partial}{\partial x_{j}}\left(\vartheta+\sigma_{k} \vartheta_{T}\right) \frac{\partial k}{\partial x_{j}} \\
& \frac{\partial \omega}{\partial t}+U_{j} \frac{\partial \omega}{\partial x_{j}}=\alpha S^{2} \\
& -\beta \omega^{2}+\frac{\partial}{\partial x_{j}}\left[\left(\vartheta+\sigma_{\omega} \vartheta_{T}\right) \frac{\partial \omega}{\partial x_{j}}\right] \\
& +2\left(1-F_{1}\right) \sigma_{\omega 2} \frac{1}{\omega} \frac{\partial k}{\partial x_{i}} \frac{\partial \omega}{\partial x_{i}} \\
& \vartheta_{T}=\frac{a_{1} k}{\max \left(a_{1} \omega, S F_{2}\right)}
\end{aligned}
$$

where $\rho$ is the density, $\mathrm{v}$ is the velocity, $\mathrm{P}$ is the pressure, $\mathrm{k}$ is the turbulent kinetic energy, $\varepsilon$ is the dissipation rate, $\omega$ is the specific dissipation rate, $\sigma_{\mathrm{k}}$, and $\sigma_{\omega}$ are the turbulent Prandtl numbers for $\mathrm{k}$ and $\omega$, a and $\beta$ are the model coefficients, $F_{2}$ is the blending function, and $\mathrm{S}$ is the source term. Steadystate solutions were implemented using the implicit algorithm. The second-degree upwind scheme was used for convective terms and the central-difference scheme was preferred for diffusion terms. Pressurevelocity coupling was analyzed using the Coupled algorithm. Convergence criteria were determined as $1 \mathrm{e}-5$ for all parameters. Initial and boundary conditions were listed in Table 3.

Table 3 Operation and boundary condition of the solution.

\begin{tabular}{|l|l|}
\hline Temperature (ambient) $[\mathrm{K}]$ & 293.15 \\
\hline Inlet temperature $[\mathrm{K}]$ & 293.15 \\
\hline Atmospheric pressure $[\mathrm{kPa}]$ & 101.325 \\
\hline Inlet pressure $[\mathrm{kPa}]$ & 410.0 \\
\hline Vacuum Pressure $[\mathrm{kPa}]$ & -50.8 \\
\hline Air film thickness $[\mu \mathrm{m}]$ & 5 \\
\hline Fluid & air \\
\hline
\end{tabular}

A hydrodynamic model is necessary to estimate the pressure drop and fluid transition from a porous material and thus to calculate the energy required to achieve the fluid transition. The pressure drop for a porous material is quantified by the DarcyForchheimer equation (Yu et al. 2006).

$$
\frac{\Delta P}{d x}=\frac{\mu}{K} v+\frac{c_{f}}{\sqrt{K}} \rho v^{2}
$$

Where $\mathrm{v}$ is the velocity in the duct, $\mathrm{K}$ permeability, $\mu$ is the fluid viscosity, $c_{f}$ Forchheimer or Ergün constant. Equation 7 can be used to qualify the pressure drop across the porous medium when appropriate values for permeability and Forchheimer coefficients are provided or vice versa. Permeability can be calculated with the following equation:

$$
K=\frac{\varepsilon^{3} D_{P}^{2}}{A(1-\varepsilon)^{2}}
$$

In Eq. (8), Dp spherical particle diameter and A are constant and it can be taken as 147 (Nakayama and Kuwahara 2000; Yu et al. 2006). The properties of the carbon porous material were listed in Table 4. Porosity, viscous resistance, and inertial resistance values were defined in the simulation (Belforte et al. 2007; Cieslicki 1994; Manocha 2003; Straatman et al. 2007; Yu et al. 2006).

The load-carrying capacity (W) of the bearing can be calculated by the surface integral of the pressure distribution of the air film (Gao et al. 2018):

$W=\iint p \cdot d S_{a}$ 
Table 4 Specifications of the carbon porous media.

\begin{tabular}{|l|l|}
\hline Viscous resistance $\left[1 / \mathrm{m}^{2}\right]$ & $5.86 \mathrm{e}+08$ \\
\hline Inertial resistance $\left[1 / \mathrm{m}^{2}\right]$ & $4.18 \mathrm{e}+04$ \\
\hline Permeability $\left[\mathrm{m}^{2}\right]$ & $1.71 \mathrm{e}-09$ \\
\hline Porosity & 0.8 \\
\hline Porous media material & carbon \\
\hline Air bearing material & aluminum \\
\hline
\end{tabular}

where $S_{a}$ is the surface area of the air film and stiffness of the air bearing (R) can be calculated using Eq. (10) (Gao et al. 2018).

$$
R=\frac{d W}{d h}=\frac{W(h+\Delta h)-W(h-\Delta h)}{2 \Delta h}
$$

where $\mathrm{h}$ is the air film thickness.

\section{RESULT AND DISCUSSIONS}

The experiments were carried out with different loads and moments. Two main experiments for compression load and tensile load were performed on each air bearing. Air film thickness (fly height) was measured after applying different loads (0-100 N) (Figs. 5, and 6) (Ruszkowski et al. 2012). With increasing applied compressive load, air film thickness values decrease however with increasing applied tensile load, air film thickness values rise.

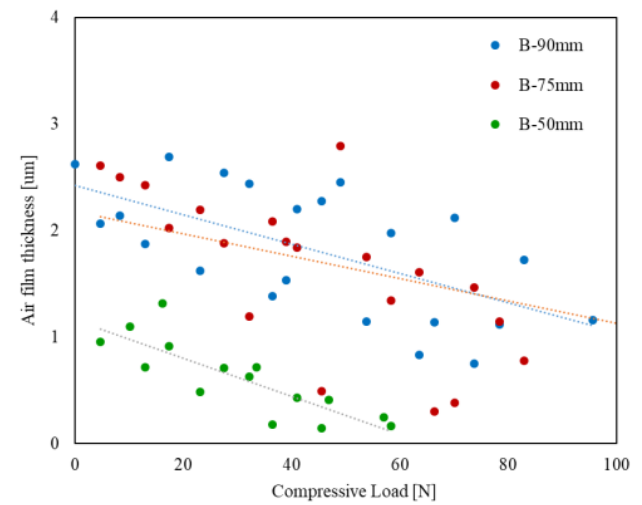

Fig. 5. Compression load - Air film thickness curve.

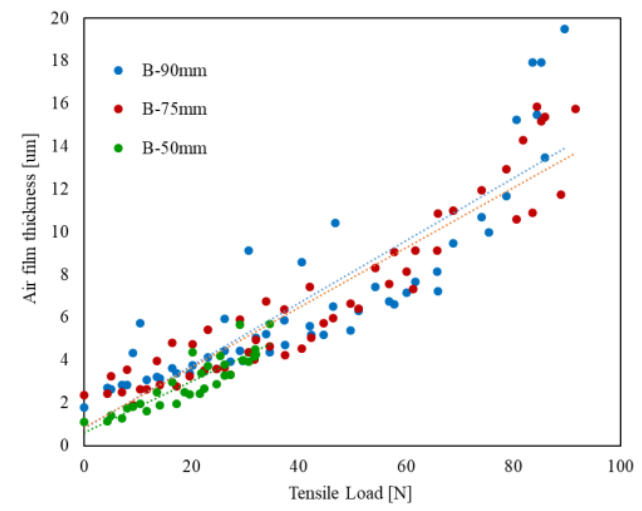

Fig. 6. Tension load - Air film thickness curve.
The measured stiffness characteristics are summarized in Table 5. Stiffness characteristics for three different bearings were measured. Although the bearing diameters are different, these results are thought to be observed since the same pressure and inlet diameters are used in the bearings.

Table 5 Summary of stiffness characteristics.

\begin{tabular}{|l|l|l|l|}
\hline Bearing & $\begin{array}{l}\Phi 90 \\
\mathrm{~mm}\end{array}$ & $\begin{array}{l}\Phi 75 \\
\mathrm{~mm}\end{array}$ & $\begin{array}{l}\Phi 50 \\
\mathrm{~mm}\end{array}$ \\
\hline $\begin{array}{l}\text { Tensile } \\
{[\mathrm{N} / \mu \mathrm{m}]}\end{array}$ & 7.7 & 12.0 & 15.3 \\
\hline $\begin{array}{l}\text { Compressive } \\
{[\mathrm{N} / \mu \mathrm{m}]}\end{array}$ & 33.3 & 25.1 & 26.0 \\
\hline $\begin{array}{l}\text { Angular } \\
{[\mathrm{Nm} / \mathrm{mrad}]}\end{array}$ & $\begin{array}{l}37.5 \\
(0-6\end{array}$ & $\begin{array}{l}27.1 \\
(0-5\end{array}$ & $\begin{array}{l}14.4 \\
(0-2 \\
\mathrm{Nm})\end{array}$ \\
\hline
\end{tabular}

According to the experiments, a correlation has been established between the load and the air film thickness (Tables 6). This correlation will be very useful in the selection and application of an air bearing and determining the characteristics of the bearing.

Table 6 Summary of tension and compression curves.

\begin{tabular}{|c|c|c|}
\hline Bearing & Tension & Compression \\
\hline$\Phi 90 \mathrm{~mm}$ & $h=-0.1157 F+$ & $h=-0.017 F+$ \\
& 5.487 & 3.103 \\
\hline$\Phi 75 \mathrm{~mm}$ & $h=-0.0744 F+$ & $h=-0.019 F+$ \\
& 4.502 & 3.098 \\
\hline$\Phi 50 \mathrm{~mm}$ & $h=-0.0600 F+$ & $h=-0.028 F+$ \\
& 2.836 & 2.256 \\
\hline
\end{tabular}

CFD simulations were carried out with experimental inputs, after designing air bearings and completing mesh independency studies. When the solution reaches a steady-state condition, the pressure distributions formed on the bottom of the bearings are given in Figs. 7, 8, and 9 for the three air bearings. Due to the vacuum input on the inside, a low-pressure zone is formed at the center while the outside is a high-pressure zone. The maximum pressure was measured at 180,141 , and $190 \mathrm{kPa}$ for the air bearings with $90 \mathrm{~mm}, 75 \mathrm{~mm}$, and $50 \mathrm{~mm}$ diameter, respectively. This pressure enables the bearing to move without friction. It also indicates the load-bearing capacity of the air bearings.

The pressure distribution of the $90 \mathrm{~mm}$ air bearing is illustrated in Figs. 7a and 7b. Since there is only one air inlet, the pressure in this region goes up to $180 \mathrm{kPa}$. But due to the porous material, the relatively homogeneous pressure distribution is observed at the bottom side of the bearing. In the porous part, the pressure ranges from 140 to 180 $\mathrm{kPa}$ (Fig. 7a). Because of the vacuum in the central part, a pressure distribution ranging from 90 to 100 $\mathrm{kPa}$ occurs (Fig. 7b). While the central part increases the stiffness of the air bearing, the outer part allows the bearing to work without contact. The 
K. Ermis and R. Sener / JAFM, Vol. 14, No. 5, pp. 1447-1457, 2021.

smaller the diameter, the more homogeneous the pressure distribution on the sole. At the bottom high-pressure zone of the $75 \mathrm{~mm}$ diameter bearing the pressure ranges from 140 to $130 \mathrm{kPa}$ (Fig. 8a).
The difference between the highest pressure and the lowest pressure is about $10 \mathrm{kPa}$. In the center, the pressure reaches $110 \mathrm{kPa}$ (Fig. 8b).

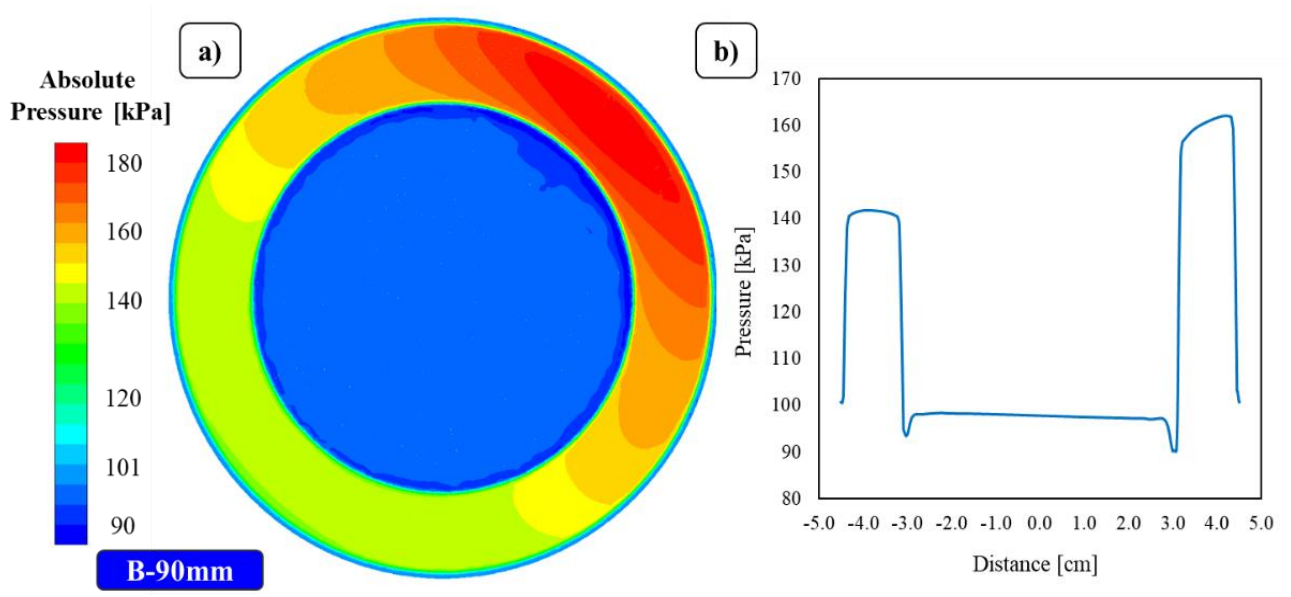

Fig. 7. (a) Pressure distribution of the air film and (b) pressure distribution in the center vertical direction for the air bearing with a diameter of $90 \mathrm{~mm}$.

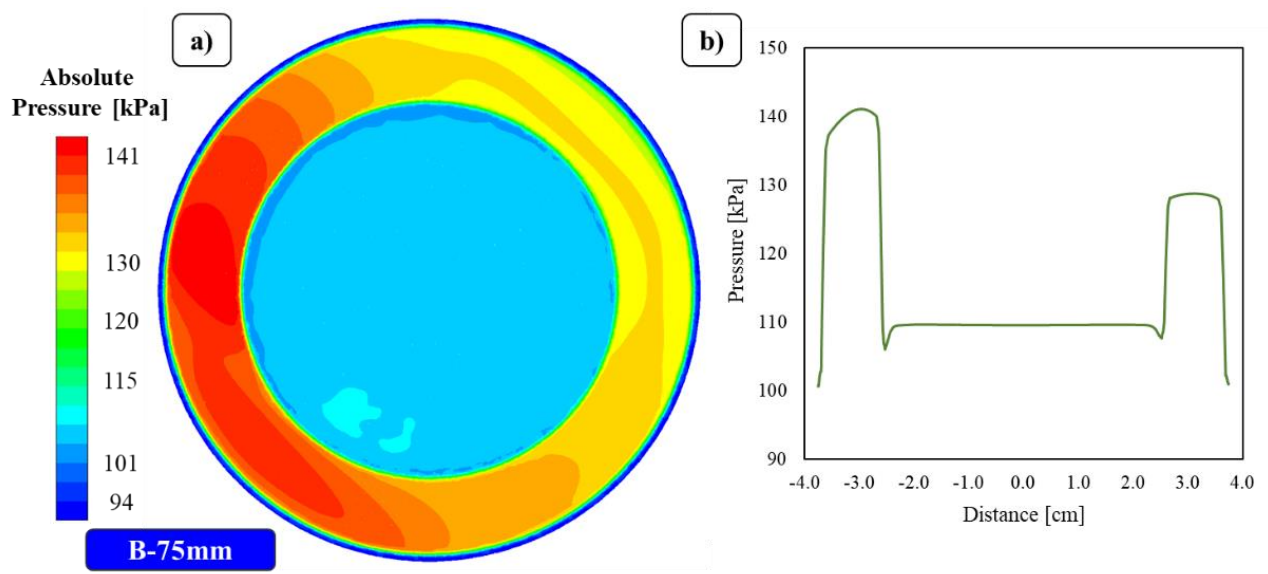

Fig. 8. (a) Pressure distribution of the air film and (b) pressure distribution in the center vertical direction for the air bearing with a diameter of $75 \mathrm{~mm}$.

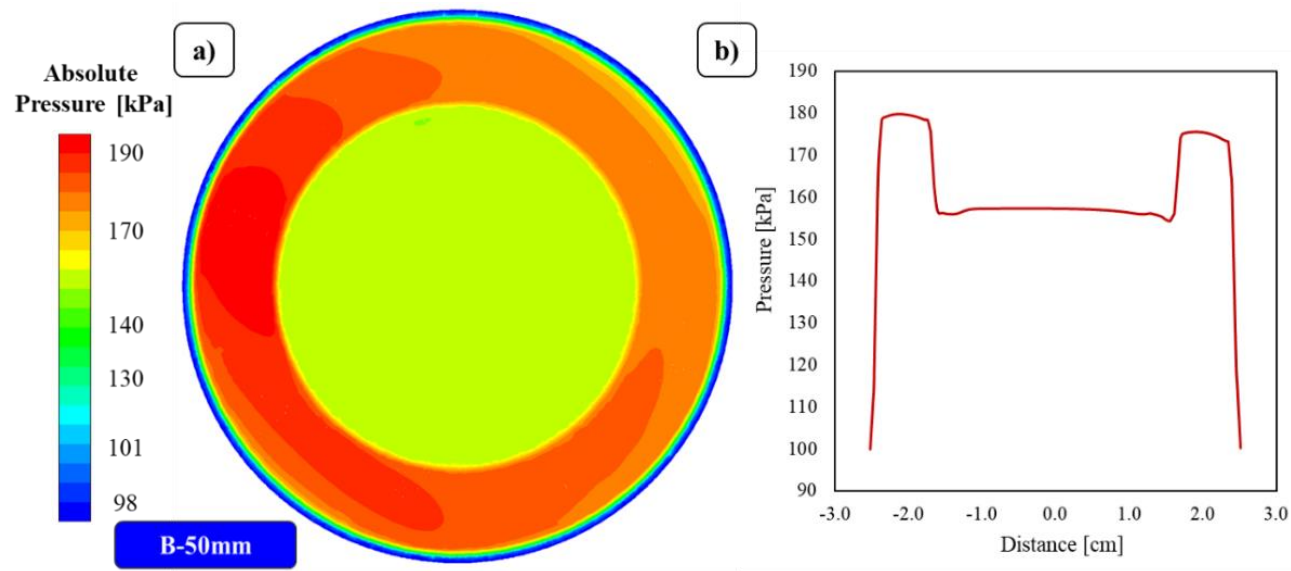

Fig. 9. a) Pressure distribution of the air film b) pressure distribution in the center vertical direction for the air bearing with a diameter of $50 \mathrm{~mm}$. 
In the air bearing with the smallest diameter, the pressure in the bottom outer side is about $180 \mathrm{kPa}$, while in the pressure at the center is about $155 \mathrm{kPa}$ (Figs. 9a and 9b). This may affect the stiffness negatively during the operation. Using lower vacuum pressure in smaller diameter air bearings can eliminate this situation.

Figure 10 shows the velocity distribution in the A-A section of the air bearing with the $90 \mathrm{~mm}$ diameter. The pressure and vacuum inlet of this bearing are vertical to the base. This design has made the velocity distribution more homogeneous in the inlet. High velocities are seen in the air inlet and when air reaches the porous material, it slows down. Higher flow velocity was obtained in the compressed air inlet region. However, the porous material distributes the flow evenly. Flow velocity reaches $300 \mathrm{~m} / \mathrm{s}$ in the local area and it drops down to 30 $\mathrm{m} / \mathrm{s}$ while passing through the porous material. Thus, the flow is uniformly distributed at the bottom side of the $90 \mathrm{~mm}$ diameter air bearing.

In the air bearings with $75 \mathrm{~mm}$ and $50 \mathrm{~mm}$ diameter, air and vacuum inlet are applied from the sides of the bearings. Therefore, the air must make an angle of 90 degrees to reach the bottom. During this movement inside the bearing, high-speed zones and vortices are observed (Figs. 11 and 12). In regions where flow is disturbed, fluid velocities increase from 200 up to $300 \mathrm{~m} / \mathrm{s}$. These bearings have a design that disrupts the flow due to difficulties during the manufacturing process. If this design is changed to one with a more aerodynamic design, load-carrying capacity and stiffness may increase. Higher stiffness and load-carrying capacity of air bearings can be obtained with improvements to their design.

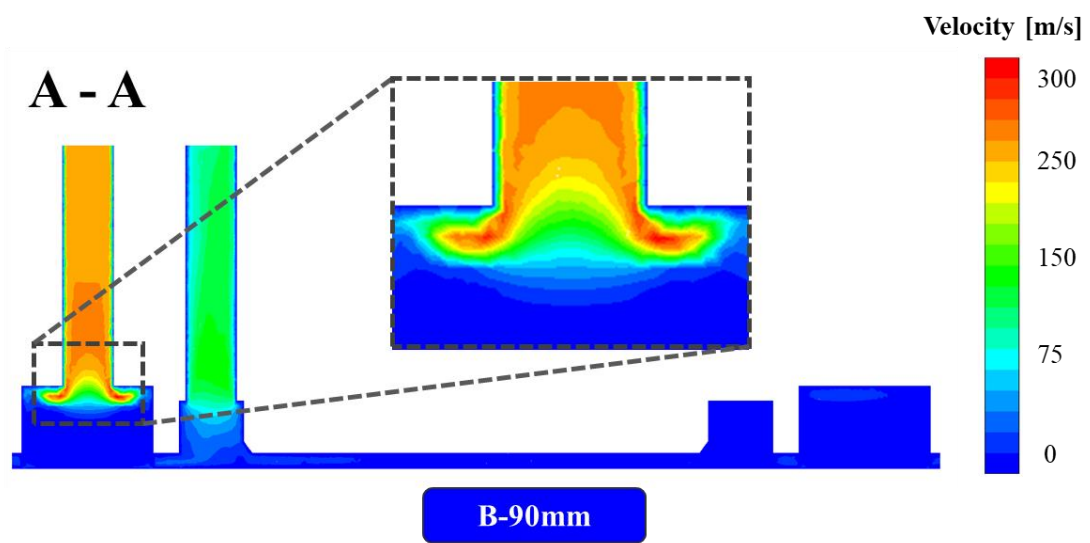

Fig. 10. Velocity distribution of air bearing with a $90 \mathrm{~mm}$ diameter in section A-A.

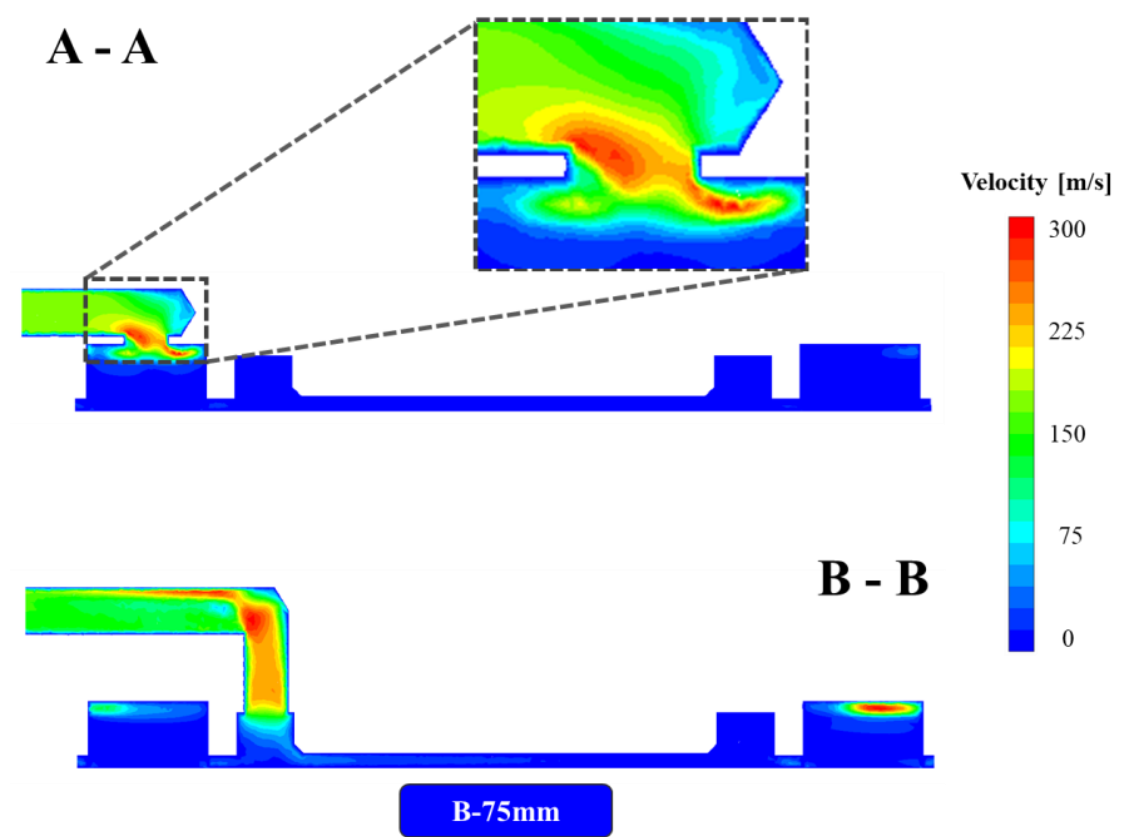

Fig. 11. Velocity distribution of the air bearing with a $75 \mathrm{~mm}$ diameter in sections A-A and B-B. 


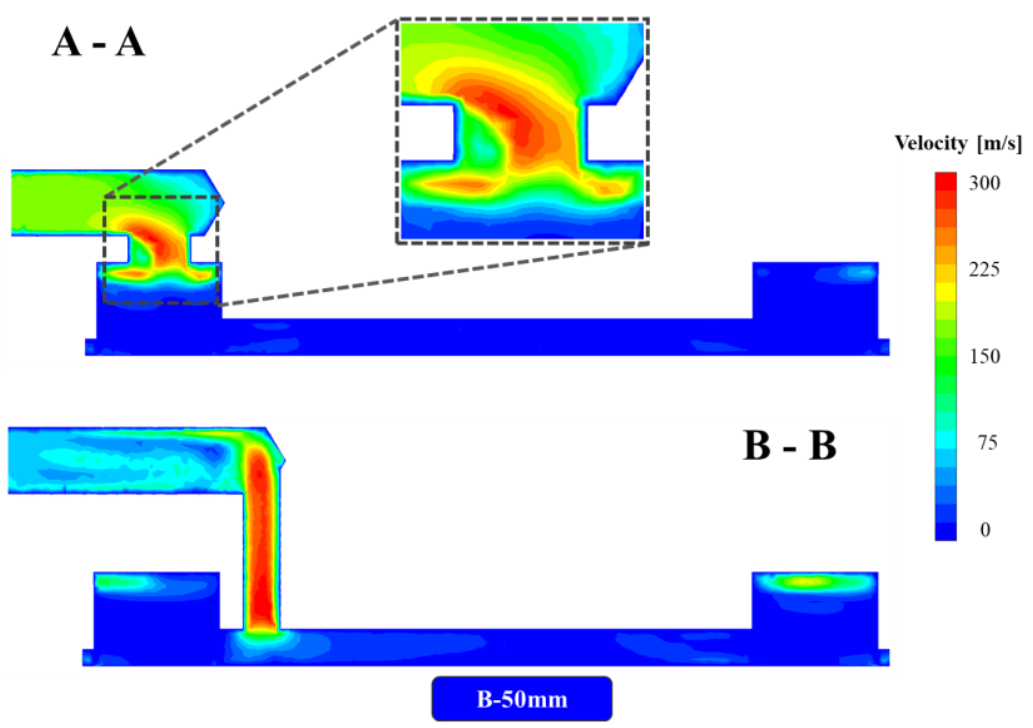

Fig. 12. Velocity distribution of the air bearing with a $50 \mathrm{~mm}$ diameter in sections A-A and B-B.

The load-carrying capacities of the air bearings were listed in Table 7 . These values were calculated using Eq. 9. It can be seen that as the diameter of the air bearings increases, their load-carrying capacity increases. Among the three air bearings, the $90 \mathrm{~mm}$ diameter air bearing has the highest load-carrying capacity at $780.45 \mathrm{~N}$. Among them, the air bearing with the lowest load-carrying capacity, specifically $324.94 \mathrm{~N}$, is the $50 \mathrm{~mm}$ diameter air bearing.

The velocity vectors inside the air bearings are shown in Fig. 13. High velocities occur at the pressure inlet and fluid velocity slows down in the porous part.

Table 7 Load-carrying capacities of air bearings.

\begin{tabular}{|c|c|}
\hline & Load-carrying capacity \\
\hline$\Phi 50 \mathrm{~mm}$ & $324.94 \mathrm{~N}$ \\
\hline$\Phi 75 \mathrm{~mm}$ & $527.35 \mathrm{~N}$ \\
\hline$\Phi 90 \mathrm{~mm}$ & $780.45 \mathrm{~N}$ \\
\hline
\end{tabular}

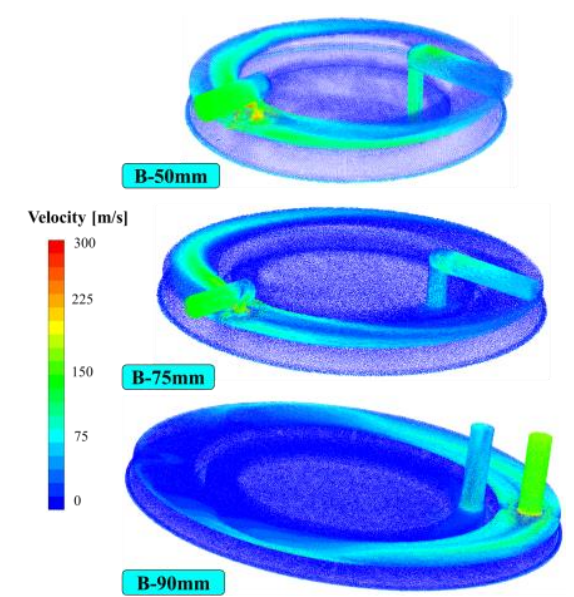

Fig. 13. Velocity vectors in the air bearings.
Air exits from the vacuum part and the sides of the bottom part. The problem of non-homogeneous distribution of airflow on the bottom side of the bearing is observed in the larger diameter bearing. This situation may cause moment formation in the bearing. Since there is an air inlet and vacuum inlet with a 90-degree elbow in the $50 \mathrm{~mm}$ and $75 \mathrm{~mm}$ diameter air bearings, flow distortions, and local high-velocity flow regions occur.

\section{CONClusion}

In the present work, numerical analysis of vacuum preloaded porous air bearings was performed. Three VPL porous air bearings with different diameters were analyzed and the results were compared. The air bearings were simulated using the CFD software. The models were generated according to the experimental conditions, characteristics, and geometries. In addition, it was determined that the model simulations were consistent with the experimental results. The main conclusions following the results of the experimental tests and the CFD studies are drawn as follows.

1. Air film thickness decreases as the compression load increases but increases as the tensile load increases for all air bearings. This increase or decrease occurs at a predictable linear rate.

2. The stiffness value increases with the lowpressure zone at the center of the air bearings. The high-pressure zone occurring at the edge procures the load-carrying capacity of the bearing and guarantees its friction-free operation.

3. The $50 \mathrm{~mm}, 75 \mathrm{~mm}$, and $90 \mathrm{~mm}$ diameter air bearings have load-carrying capacities of $324.94,527.35$, and $780.45 \mathrm{~N}$, respectively.

4. According to the CFD model, an optimization study can be carried out to maximize the stiffness and load-carrying capacity of the air 
bearings by changing working parameters and their designs.

\section{ACKNOWLEDGMENT}

The authors would like to thank Prof. Dr. Kaan Erkorkmaz, University of Waterloo, Canada for providing experimental data and information regarding air bearings for this study.

\section{REFERENCES}

Aguirre, G., F. Al-Bender and H. Van Brussel (2010). A multiphysics model for optimizing the design of active aerostatic thrust bearings. Precision Engineering 34(3), 507-515.

Al-Bender, F. and H. Van Brussel (1992). Tilt characteristics of circular centrally fed aerostatic bearings. Tribology International 25(3), 189-197.

ANSYS Inc. (2019). ANSYS Fluent Software: CFD Simulation.https://www.ansys.com/products/fl uids/ansys-fluent

Belforte, G., T. Raparelli, V. Viktorov and A. Trivella (2007). Permeability and inertial coefficients of porous media for air bearing feeding systems. Journal of Tribology 129(4), 705-711.

Bleuler, H., R. Clavel, J. M. Breguet, H. Langen and E. Pernette (2000). Issues in precision motion control and microhandling. Proceedings-IEEE International Conference on Robotics and Automation, 1(April), 959964.

Cai, T., M. Zhang, Y. Zhu and C. Hu (2011). Dynamic modeling and analysis of a 3-DOF ultra-precision positioning stage with air bearinga. Procedia Engineering 16(1), 264270.

Cieslicki, K. (1994). Investigations of the effect of inertia on flow of air through porous bearing sleeves. Wear 172(1), 73-78.

Connolly, L. G., T. F. Yao, A. Chang and M. Cullinan (2019). A tip-based metrology framework for real-time process feedback of roll-to-roll fabricated nanopatterned structures. Precision Engineering 57(1), 137-148.

Devitt, A. J. (1999). Porous vs. orifice air bearing technology. In New Way Air Bearings (Vol. 26 , Issue 6).

Fourka, M. and M. Bonis (1997). Comparison between externally pressurized gas thrust bearings with different orifice and porous feeding systems. Wear, 210(1-2), 311-317.

Gao, Q., W. Chen, L. Lu, D. Huo and K. Cheng (2019). Aerostatic bearings design and analysis with the application to precision engineering: State-of-the-art and future perspectives. Tribology International 135(February), 1-17.
Gao, Q., L. Lu, W. Chen and G. Wang (2018). Optimal design of an annular thrust air bearing using parametric computational fluid dynamics model and genetic algorithms. Proceedings of the Institution of Mechanical Engineers, Part J: Journal of Engineering Tribology 232(10), 1203-1214.

Gordon, D. J. and K. Erkorkmaz (2012). Precision control of a T-type gantry using sensor/actuator averaging and active vibration damping. Precision Engineering 36(2), 299 314.

Horiuchi, T. and Y. Suzuki, (2013). Microfabrication of air-bearing grooves onto inner surfaces of fine copper pipes. Microelectronic Engineering 110(1), 422-426.

Ishibashi, K., A. Kondo, S. Kawada, M. Miyatake, S. Yoshimoto and T. Stolarski (2019). Static and dynamic characteristics of a downsized aerostatic circular thrust bearing with a single feed hole. Precision Engineering 60(September), 448-457.

Kakaç, S., A. Pramuanjaroenkij and X. Y. Zhou (2007). A review of numerical modeling of solid oxide fuel cells. In International Journal of Hydrogen Energy 32(7), 761-786.

Khim, G. and C. H. Park, (2013). Analysis on the Static Performance of Vacuum-Preloaded Porous Air Bearings. Journal of the Korean Society for Precision Engineering 30(12), 1327-1333.

Kwan, Y. B. P. and J. Corbett (1998). Porous aerostatic bearings-an updated review. Wear, 222(2), 69-73.

Li, Y. F., Y. H. Yin, H. Yang, X. E. Liu, J. Mo and H. L. Cui (2018). Micro-vibration analysis and optimization of aerostatic bearing with pocketed orifice-type restrictor. Journal of Applied Fluid Mechanics 11(4), 1115-1124.

Liu, J. (2020). A dynamic modelling method of a rotor-roller bearing-housing system with a localized fault including the additional excitation zone. Journal of Sound and Vibration 469, 115144.

Liu, J. and Y. Shao (2018). An improved analytical model for a lubricated roller bearing including a localized defect with different edge shapes. Journal of Vibration and Control 24(17), 3894-3907.

Manocha, S. M. (2003). Porous carbons. Sadhana Academy Proceedings in Engineering Sciences 28(1-2), 335-348.

Miyatake, M. and S. Yoshimoto (2010). Numerical investigation of static and dynamic characteristics of aerostatic thrust bearings with small feed holes. Tribology International 43(8), 1353-1359.

Nakayama, A. and F. Kuwahara (2000). Numerical Modeling of Convective Heat Transfer in 
K. Ermis and R. Sener / JAFM, Vol. 14, No. 5, pp. 1447-1457, 2021.

Porous Media Using Microscopic Structures. In Handbook of Porous Media, Second Edition.

New Way Air Bearings (2006). Air Bearing Application and Design Guide. www.newwayairbearings.com

Nishio, U., K. Somaya and S. Yoshimoto, (2011). Numerical calculation and experimental verification of static and dynamic characteristics of aerostatic thrust bearings with small feedholes. Tribology International 44(12), 1790-1795.

Oiwa, N., M. Masuda, T. Hirayama, T. Matsuoka and H. Yabe, (2012). Deformation and flying height orbit of glass sheets on aerostatic porous bearing guides. Tribology International 48, 2 7.

Okyay, A., K. Erkorkmaz and M. B. Khamesee, (2018). Mechatronic design, actuator optimization, and control of a long stroke linear nano-positioner. Precision Engineering 52(1), 308-322.

Plante, J. S., J. Vogan, T. El-Aguizy and A. H. Slocum (2005). A design model for circular porous air bearings using the 1D generalized flow method. Precision Engineering 29(3), 336-346.

Ruszkowski, A., K. Erkorkmaz and G. Stubley (2012). Characterization of vertical and pitch stiffnesses of porous vacuum preloaded air bearings. Proceedings - ASPE 2012 Annual Meeting.

Rybus, T. and K. Seweryn (2016). Planar airbearing microgravity simulators: Review of applications, existing solutions and design parameters. Acta Astronautica 120(1), 239259.

Schenk, C., S. Buschmann, S. Risse, R. Eberhardt and A. Tünnermann (2008). Comparison between flat aerostatic gas-bearing pads with orifice and porous feedings at high-vacuum conditions. Precision Engineering 32(4), 319328.

Shi, J., H. Cao and H. Jin (2019). Investigation on the static and dynamic characteristics of 3
DOF aerostatic thrust bearings with orifice restrictor. Tribology International 138(April), 435-449.

Singh, K. C. and N. S. Rao (1982). Analysis of aerostatic porous annular thrust bearings with tilt. Wear 80(3), 291-299.

Slocum, A., M. Basaran, R. Cortesi and A. J. Hart (2003). Linear motion carriage with aerostatic bearings preloaded by inclined iron core linear electric motor. Precision Engineering 27(4), 382-394.

Straatman, A. G., N. C. Gallego, G. Yu and B. E. Thompson (2007). Characterization of porous carbon foam as a material for compact recuperators. Journal of Engineering for Gas Turbines and Power 129(2), 326-330.

Torralba, M., M. Valenzuela, J. A. Yagüe-Fabra, J. A. Albajez and J. J. Aguilar (2016). Large range nanopositioning stage design: A threelayer and two-stage platform. Measurement: Journal of the International Measurement Confederation 89, 55-71.

Wardle, F. (2015). Ultra-Precision Bearings. In Woodhead Publishing (1. Edition). Woodhead Publishing.

Xue, R., X. Zheng, L. Yue, S. Zhang and C. Weng (2020). Numerical study on supersonic boundary-layer transition and wall skin friction reduction induced by fuel wall-jet combustion. Acta Astronautica 174(March), 11-23.

Ye, Y., X. Chen and X. Luo (2009). Dynamic characteristics of aerostatic bearings in nanoprecision stage. 2009 International Conference on Information and Automation 2003, 10501055.

Yoshimoto, S. and K. Kohno (2001). Static and dynamic characteristics of aerostatic circular porous thrust bearings (effect of the shape of the air supply area). Journal of Tribology 123(3), 501-508.

Yu, Q., B. E. Thompson and A. G. Straatman (2006). A unit cube-based model for heat transfer and fluid flow in porous carbon foam. Journal of Heat Transfer 128(4), 352-360. 